\title{
Functionalized microneedles for continuous glucose monitoring
}

\author{
Kai Takeuchi and Beomjoon Kim * (1)
}

\begin{abstract}
Microneedles (MNs) have been established as promising medical devices as they are minimally invasive, cause less pain, and can be utilized for self-administration of drugs by patients. There has been rapid development in MNs for transdermal monitoring and diagnostic systems, following the active research on fabrication methods and applications for drug delivery. In this paper, recent investigations on bio-sensing using MNs are reviewed in terms of the applicability to continuous glucose monitoring system (CGMS), which is one of the main research focuses of medical engineering technologies. The trend of the functionalized MNs can be categorized as follows: (i) as a sensing probe, and (ii) as a biological fluid collector. MNs as in vivo sensors are mainly integrated or coated with conductive materials to have the function as electrodes. MNs as fluid collectors are given a certain geometrical design, such as a hollow and porous structure aided by a capillary action or negative pressure, to extract the interstitial fluids or blood for ex vivo analysis. For realization of CGMS with MNs, a long-term accurate measurement by the MN-based sensing probe or a fluidic connection between the MN-based fluid collector and the existing microfluidic measurement systems should be investigated.
\end{abstract}

Keywords: Microneedles, Glucose sensor, Interstitial fluids

\section{Introduction}

Diagnostic systems using bio-sensing technologies have been the main focus of research for the last few decades owing to the high demand for more rapid, precise, and easy-to-use applications for the well-being of society. One of the aims in the development of recent diagnostic devices is to achieve a shorter time period of analysis with a reduced amount of the required biological samples by microfluidic and electrochemical components realized by new fabrication technologies, such as the CMOS and MEMS. However, in spite of the advantages of such diagnostic systems, called micro total analysis systems (pTASs), the methodologies of body fluid sampling is limited to conventional methods such as hypodermic needles.

Conventional needles have several disadvantages such as the associated unavoidable pain, and requirement of a high level of skilled training of the medical professionals,

*Correspondence: bjoonkim@iis.u-tokyo.ac.jp

Institute of Industrial Science, The University of Tokyo, 4-6-1 Komaba, Meguro-ku, Tokyo 153-8505, Japan even though they extract too much amount of the body fluids for pTASs. Hence, microneedles (MNs) have attracted significant attention as next-generation devices to access biological tissues through the skin without pain due to their microsized dimension, e.g. typically a length of less $1 \mathrm{~mm}$ and diameter of less $500 \mathrm{pm}$. Subsequent to the reports of MNs being novel tools to penetrate the skin and to deliver drugs and vaccines with a minimal invasion and pain, a wide range of research has focused on the methods of their fabrication, supported by micromachining technologies and the applications for transdermal, self-administration of drugs [1-12].

In addition to the medical drug delivery, MNs, e.g., dissolvable MN patches, are also well known as commercialized cosmetic products for skin treatments [13, 14] nowadays, enabling an efficient localized drug delivery process, self-administered by the patient, with less fear of skin penetration [15].

Of late, MNs are expected to be applied to the extraction of biological information out of human skin in contrast to drug delivery into the skin through microsized pores. The targets of MN bio-sensing are blood or 
interstitial fluids (ISFs), as the MNs can reach a depth of approximately $1 \mathrm{~mm}$ where the vessels are located and ISF surrounds the cells in the epidermis. The analysis methodologies of such biological fluids are well developed with the advancement of both enzymatic and nonenzymatic electrochemical science for the detection and measurement of medical biomarkers such as metabolites, ions, proteins, and glucose. In the field of MN-based diagnostic systems, ISF is the main target sample since ISF contains various valuable biomarkers such as $\mathrm{K}^{+}$and $\mathrm{Na}^{+}$ions, nitrogen oxide, and glucose, the analysis, as well as that of blood [16].

In particular, the glucose measurement is the focus of research on the bio-sensing in accordance with societal demands, as Type 1 and 2 diabetes are, nowadays, one of the most serious diseases due to its long-term effects on the health of patients, the large number of patients involved, and the huge amount of social and economical expenses. Currently, the blood glucose level is monitored by self-monitoring blood glucose (SMBG) devices in which the patients are required to prick their fingers to extract a drop of blood 2-5 times a day for colorimetric or enzymatic electrochemical measurements, which results in a physical and mental strain on the patients [17].

Furthermore, the concept of continuous glucose monitoring system (CGMS) has been initiated as a more efficient methodology to control the blood glucose level for prevention, diagnosis, and treatment of diabetes. CGMS allows a seamless observation of a change in the blood glucose level, even when the patients are not able to measure the glucose level by themselves [18-21]. However, the process of monitoring is carried out by conventional needles that restrict the activities of the patients, as certain movements of the body may cause some pain or dislodge the implanted needle.

For these reasons, monitoring of the blood glucose level is the main objective of research in MN-based biosensing, due to its applicability to point-of-care or continuous diagnostic devices without pain. Even though ISF reflects the change in the blood glucose level after a 4-10 min delay [22], it is still the target of the MN-based glucose sensors, so as to avoid significant pain by the penetration. The basic strategy of the MN-based glucose sensor is to utilize the amperometric measurement with immobilized glucose oxidase $\left(\mathrm{GO}_{\mathrm{x}}\right)$ for the detection of $\mathrm{H}_{2} \mathrm{O}_{2}$ generated by the reaction given below:

$$
\text { Glucose }+\mathrm{O}_{2} \stackrel{\text { GOx }}{\longrightarrow} \text { Gluconic acid }+\mathrm{H}_{2} \mathrm{O}_{2} \text {. }
$$

The generated $\mathrm{H}_{2} \mathrm{O}_{2}$ is detected by a working electrode (WE), following the re-action given below [23]:

$$
\mathrm{H}_{2} \mathrm{O}_{2} \stackrel{+700 \mathrm{mV}}{\longrightarrow} \mathrm{O}_{2}+2 \mathrm{H}^{+}+2 \mathrm{e}^{-} \text {. }
$$

In addition to the amperometric measurement, potentiometric and optical methods such as enzyme field effect transistor (FET)-based [24, 25], ion selective field effect transistor (ISFET)-based [26, 27], and infrared (IR) lightbased measurements $[28,29]$ have been widely explored. Thus, in order to develop clinically diagnostic systems using MNs, the MNs should have specific functions according to those methodologies, in addition to their own capability of penetrating the skin.

On the basis of this concept, MNs have been integrated with a variety of measurement systems by a specialized design of the material of $\mathrm{MN}$, its configuration, and structure. These studies are emerging rapidly from the level of laboratory to clinical tests. Therefore, even though there are excellent review papers already available on bio-sensing using MNs [30-32], it is necessary to summarize the present-day investigations to have a full picture of the research conducted so far on MN-based glucose sensors, especially CGMS.

In this paper, a review of the recent progress on the MN devices functionalized for diagnostic systems, especially glucose monitoring, is presented. The functionally developed MNs for diagnostic bio-sensing are categorized into MNs as (i) sensing probes and (ii) biological fluids collectors, according to their applications. Those functionalized MNs are discussed from the perspective of the applicability for CGMS devices.

\section{MNs as sensing probes}

\subsection{MNs coated with conductive materials}

Miniaturization of a sensing probe to form an MN configuration is a natural way for minimally invasive medical monitoring, as successful results on the detection and measurement of biomarkers such as glucose were achieved by using implantable biosensors [21,33]. As the scientific methodology for the measurement of analytes in ISF using electrochemical components has been highly developed, it is justifiable to functionalize MNs as in vivo sensing probes beneath the skin, enabling point-of-care measurement owing to their easy penetration into the skin. For this, MNs should be combined with conductive materials as electrodes to connect electrochemical reactions in the body and external amperometric or potentiometric measurement systems.

One of the methods to provide MNs electric function is to coat the $\mathrm{MN}$ with electrochemical components. As a result of the development in micro fabrication technology, MN configurations of biocompatible materials are typically achieved using relatively simple methodologies such as mold casting [34-37] and drawing lithography $[38,39]$. However, these methods are not suitable for conductive materials including metals compared to removal machining including MEMS processes. Furthermore, an 
electrochemical interface should be realized at the interface between MN-based electrodes and biological fluids. For this reason, many studies focus on the coating of $\mathrm{MNs}$ with conductive materials represented by $\mathrm{Au}, \mathrm{Pt}$, and $\mathrm{Ag} / \mathrm{AgCl}$ as the WEs, counter electrodes (CEs), and reference electrodes (REs), respectively, in order to enable an MN to function as an electrochemical electrode. In this methodology, metal [40-46], epoxy [47-49], SU-8 [50-53], and polymer [53-59] MNs are coated with metal layers using electroplating or vapor deposition. The covering metal layers are connected to the extract electrodes and the measurement system to conduct the input and output signals.

MNs coated with conductive materials to be used as glucose sensors have in been realized mainly by using the immobilized $\mathrm{GO}_{\mathrm{x}}$ on the surface of the $\mathrm{MN}[44,46$, $51-53,55-58,60]$. The Cass's group developed the polycarbonate [60] and epoxy-based MN array [52] coated with $\mathrm{Pt}$ as WE and $\mathrm{CE}$, and $\mathrm{Ag} / \mathrm{AgCl}$ as the reference electrodes for CGMS application. The MN array was designed with three separate electrode areas and fabricated by the mold casting method, as shown in Fig. 1a. This coated MN-based glucose sensor is developed with the measuring setup enabling long-term glucose monitoring in a mobile and wearable system. The polycarbonate-based $\mathrm{MN}$ array was evaluated for its performance in Type 1 diabetes patients for $24 \mathrm{~h}$ after conducting safety tests on healthy human bodies for 6 and $24 \mathrm{~h}$. The result shows the feasible accuracy of the glucose monitoring for Type 1 diabetes, as described in Fig. $1 \mathrm{~b}$.

\subsection{MNs integrated with electrodes}

Another approach to devise an $\mathrm{MN}$ as a sensing probe is to integrate a MN-based mechanical guide with the micro-sized electrodes. The non-MN shaped electrodes are integrated with the MNs by being enclosed in the hollow MNs [61-66]. In this method, the hollow MNs work as mechanical guides for the enclosed conductor (e.g. carbon paste) to puncture the skin and support the electrodes inside the MNs because, as mentioned above, the electrode materials are not suitable for the MN fabrication by a simple process. This approach provides the advantage that the immobilized enzyme and the electrodes are protected by the surrounding hollow structure of MNs during the penetration into the skin and in the measurement process.

On the contrary to the hollow MNs enclosing conductive components, a few studies focused on the fabrication of electrodes on in-plane probes, which is obtained from a Si wafer by CMOS and MEMS-based process [66, 67]. These $\mathrm{MN}$-shaped sensing probes can be likened to conventional sensing probes for neural

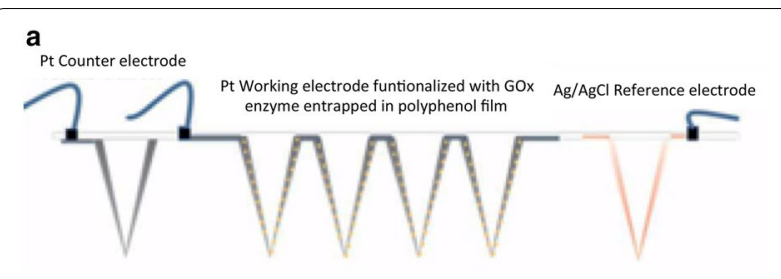

b

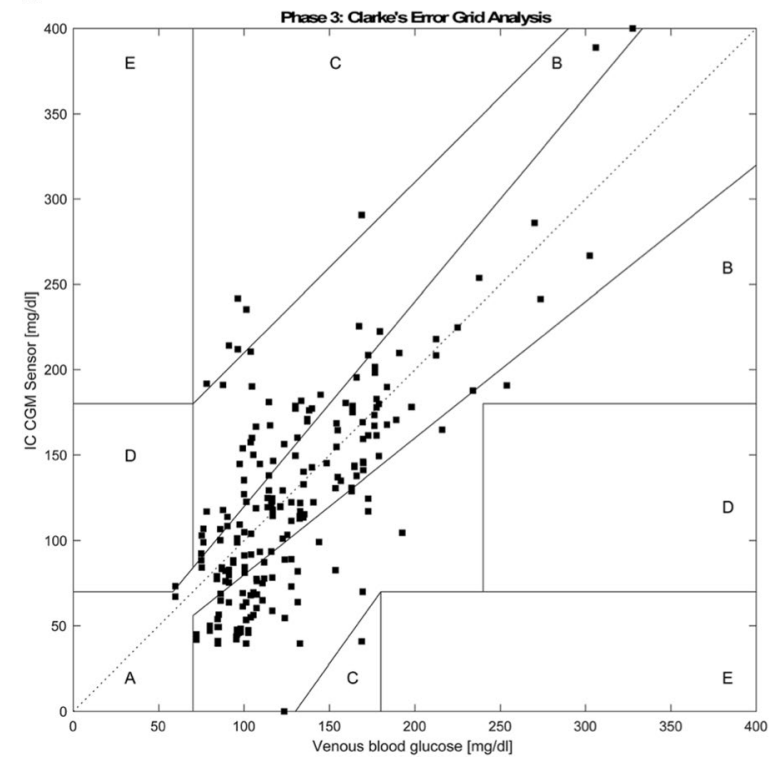

Fig. 1 a Schematic of the coated MN-based electrodes, b Measurement of Clarke error in the $24 \mathrm{~h}$ clinical test of the glucose monitoring system with the MN array (Reproduced with permission [52]. Copyright 2018, the Royal Society of Chemistry)

signal measurement [68-71]. In this approach, gold or platinum electrodes with circuits and contact pads are fabricated on in-plane micro-sized probes, which are provided a configuration of MNs or assembled with hollow MNs. Although this concept leads to a lesser number of MNs compared to out-of-plane MN arrays in accordance with the fabrication principle, CMOSbased probes are relatively suitable for integration with the setup of the measurement system compared to other MN-based sensors.

Ribet et al. reported the fabrication of a Si-based hollow MN with side openings that encloses the platinum WE and CE and the iridium oxide RE on a microsized probe fabricated by the CMOS processes (Fig. 2a) [66]. In this approach, ISF permeates the side openings and is sequentially measured by the three electrodes enclosed in the hollow MN, as illustrated in Fig. 2b, c. The device successfully detects the amperometric signal according to the given glucose concentration even though only short time measurement is performed, as shown in Fig. 2d. 


\section{a}

(I)

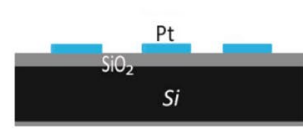

(III)

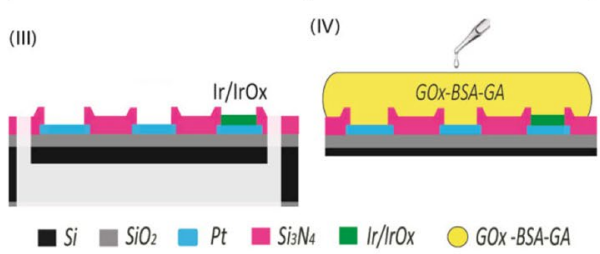

C
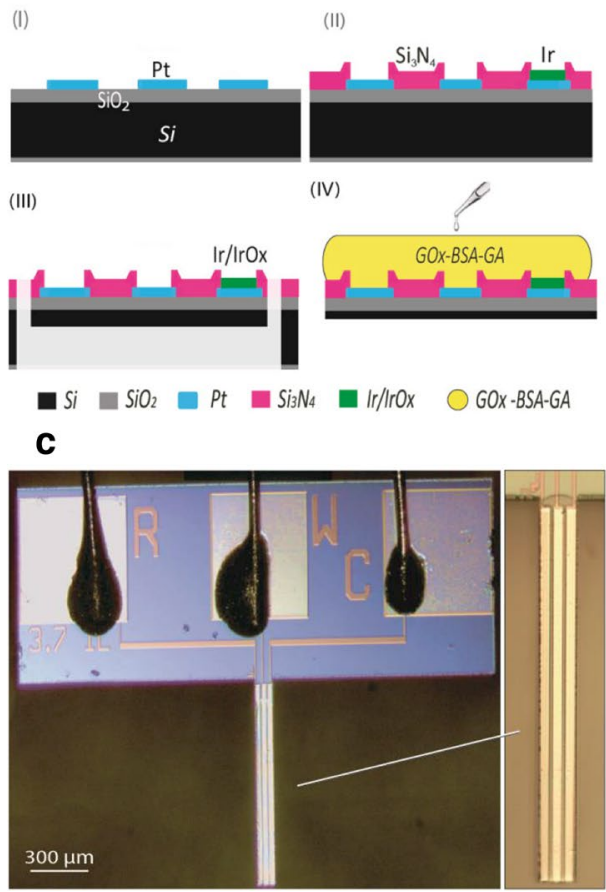

(IV)

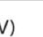

b

a

d

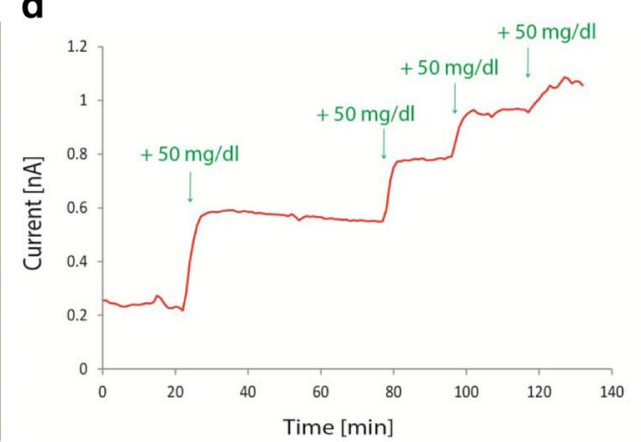

Fig. 2 a Cross-sectional fabrication process of the electrodes on the Si MN. b An optical image of the assembled hollow MN and measurement setups. SEM image of the hollow MN is shown on right hand, c A micrograph of the fabricated electrodes on the Si probe, $\mathbf{d}$ Amperometric measurement of glucose by the hollow MN-based sensor (Reproduced with permission [66]. Copyright 2018, IEEE)

\section{MNs as a biological fluid collectors}

\subsection{Hollow MNs}

An alternative way to access biological information is to extract body fluids through the micro pores punctured by the MNs. In this category, the MNs are defined as biological fluid collectors creating a transdermal fluidic path at the interface between the inner tissue including the dermis and the outer part of the skin. The MNs collecting biological fluids such as blood and ISF have attracted the interest of researchers as they have the potential to be utilized in the existing ex vivo diagnostic systems including pTAS devices. However, it is necessary for the MNbased collector to collect at least a few $\mathrm{pL}$ of liquid for a reliable measurement of the analytes [72-74], whereas the MN-based sensing probes have a high availability of biological fluids under the surface of the skin. Therefore, the sampling performance is critical to the MN-based fluid collectors.

Research on the application of hollow MNs for the collection of biological fluid was initiated earlier than that of other types of MNs, probably due to their suitability to the sampling of a sufficient amount of the biological fluid in a manner similar to conventional hypodermic needles. The hollow MNs extract ISF or blood into their bores by a capillary action or external negative pressure.
As the hollow MNs were put to use as micro injection needles for localized drug delivery into the skin at the initial stage of the development of research in MNs [3, 75], the methods of fabrication and theoretical analysis of the hollow MNs are well investigated [4, 76-81]. As a result, the hollow MNs for fluid extraction are mainly made of $\mathrm{Si}$ or metals using MEMS-based micromachining technologies, including chemical etching or electroplating. In addition, bores in hollow MNs need to be precisely aligned on inlets of fluidic measurement systems to collect biological fluids for a rapid analysis. According to this strategy, hollow MN-based sensing systems are highly developed for the measurement of protein [82], ions [83], glucose [84-90], and other analytes [91-93] in either blood or ISF.

The Jung's group presented a vacuum-pressure aided blood extraction system with Ni hollow MN for a colorimetric glucose sensor [89]. They connected the hollow MN fabricated by electroplating to a PDMS micro chamber, which is elastically deformable by a finger push to evacuate the air inside, as illustrated in Fig. 3a. The manually generated negative pressure drives the blood from the vessel into the chamber via the hollow $\mathrm{MN}$, followed by a capillary transport of the blood through a filtering and $\mathrm{GO}_{\mathrm{x}}$-immobilized paper channel. The glucose level 

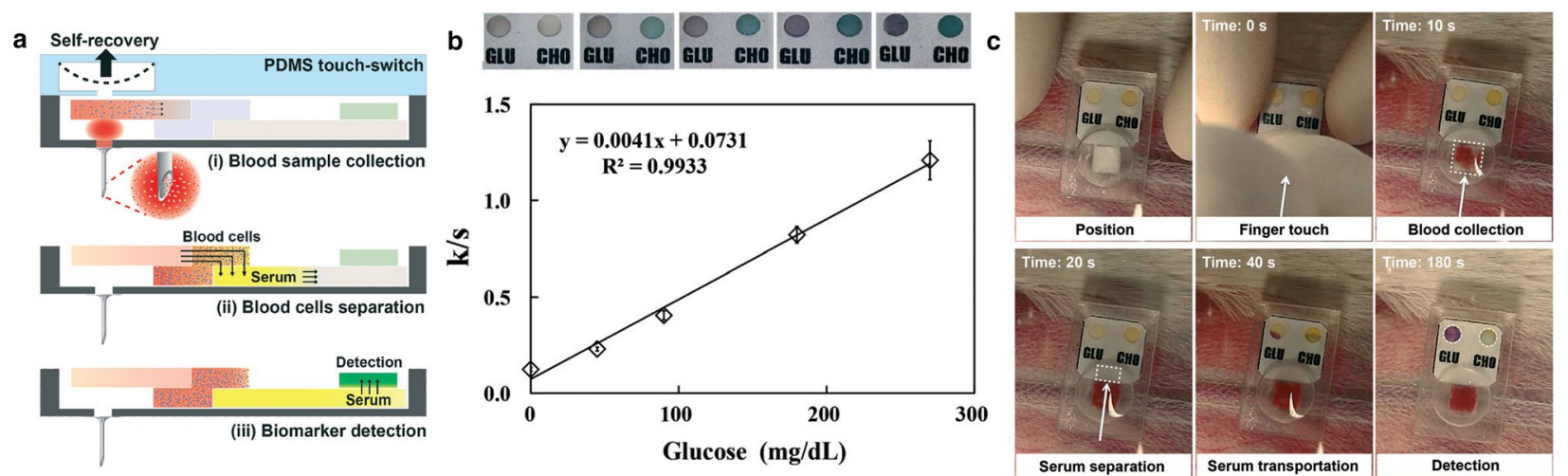

Fig. 3 a The sampling and measurement process of blood using a hollow MN and paper-based sensor. b The change of color (above) and calibration curves for glucose measurement (below). c A series of optical images of the blood sampling and glucose measurement in a rabbit ear with the integrated hollow MN and the paper-based sensor (Reproduced with permission [89]. Copyright 2015, the Royal Society of Chemistry)

is observed by comparing the colorimetric change of the paper (Fig. 3b), which results in a blood glucose measurement of a rabbit ear (Fig. 3c). In terms of point-of-care glucose sensors, the hollow-MN based measurement system showed the potential to be applied for clinical use as a miniaturized system of the present glucose monitoring kit using finger pricks and colorimetric reactions.

\subsection{Swelling MNs}

An alternative method to the collection of ISF by using an $\mathrm{MN}$ array is to employ swelling materials for the body for the MN. It has been demonstrated that hydrogel MNs are capable to extract ISF by swelling, which results in a fluidic conduit [94-97], as well as to release the drug contained in the MN matrix at the same time [98-101].

ISF is absorbed in the dry hydrogel MNs by diffusion, after their penetration into the skin, which has to be followed by the process of separation of the sampled ISF from the MN array, by using centrifugation, either with or without immersion into a solvent. Although the swelling MNs intrinsically need the additional process to remove the analytes, the main fabrication process by mold casting is relatively simple and they have the ability to collect ISF efficiently due to the relatively large capacity of the sampling volume. This approach is also applied to the measurement of glucose levels $[96,97]$.

\subsection{Porous MNs}

Although the porous $\mathrm{MNs}$ were widely considered as drug delivery devices [34, 102-106], the porous $\mathrm{MN}$ array as an ISF collector has only been developed in recent years [107-110]. Porous MNs have the capability to absorb ISF by a capillary action depending on the geometry of pores in MN bodies and their hydrophilicities. Although the concept of ISF extraction by the porous MNs has been proposed before, conventional porous MNs need to be centrifuged or dipped in a solvent to separate the absorbed ISF, in the same manner as in the case of the swelled MNs, which leads to an increase in the number of steps involved in analysis.

The first successful study of a porous MN array for ISF collection and a fluidic connection to a external measurement system was reported by the Nishizawa's group $[109,110]$. As the efficiency of collection by the capillary action into the porous MNs is rated lower than that by the hollow MNs [111], the present study employs the porous $\mathrm{MN}$ array only as a transdermal fluidic path and not for the direct outward flow of ISF, as illustrated in Fig. 4a. The absorbed ISF in the polyethylene glycol (PEG) MN array (Fig. 4b) was fluidically connected to a hydrogel located on the rear side of the MN array, so that the electrolytes conduct current from electrodes to the dermis through the porous structure. Figure $4 \mathrm{c}$ describes the schematic of the measurement of the DC electric resistance on human skin, whose results showed the detection of an intercellular swelling according to the change of distance between two porous $\mathrm{MN}$ array on a leg skin, as illustrated in Fig. 4d. Even though the target of the measurement was not glucose but electrolytes in ISF in this report, the transdermal fluidic path created by the porous MN array made of biodegradable materials such as PEG is very promising for CGMS. In addition to this approach, Takeuchi et al. reported the fluidic connection between a porous structure and a microfluidic channel network fabricated by a MEMS process [112].

\section{Towards CGMS using MNs}

Considering the research history of the micro-sized glucose sensors, MNs functionalized for bio-sensing systems are comparatively recent. Hence, there are still challenges 

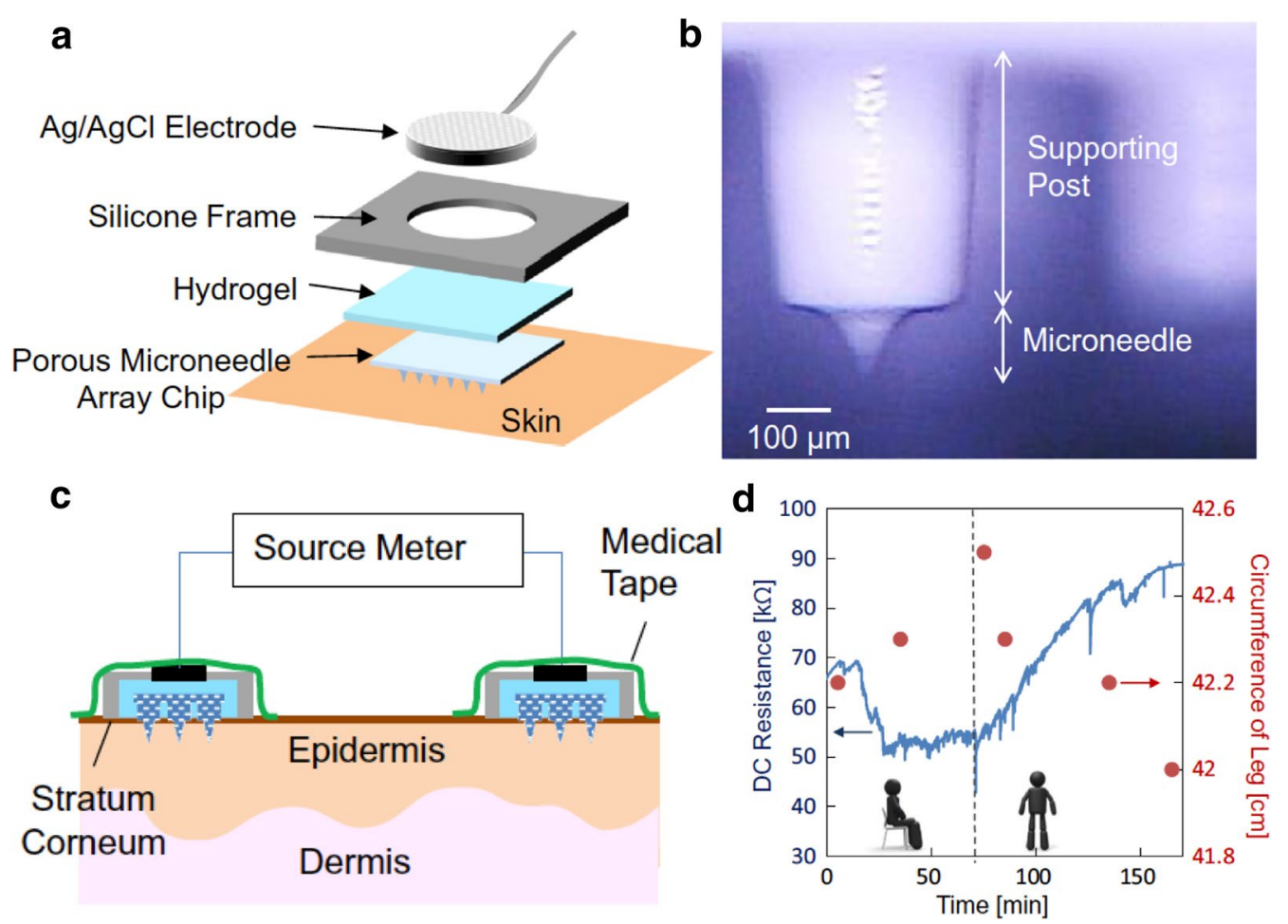

Fig. 4 a Schematic of the porous MN-based intercellular swelling measurement system. b A micrograph of the porous PEG MN. c Schematic of the DC resistance measurement by two porous $\mathrm{MN}$ array. $\mathbf{d}$ The $\mathrm{DC}$ resistance measured by the porous $\mathrm{MN}$ array and the circumference changed by a movement (Reproduced with permission [1 10]. Copyright 2015, Springer Science + Business Media, LLC)

to be faced in the development of MN-based bio-sensing devices, especially for CGMS.

As discussed above, the MN-based electrodes, especially MNs coated with conductive layers, have been successfully indicated to monitor the blood glucose level continuously. This is because the MN-based electrodes are in direct contact with a sufficient amount of fluids beneath the skin for a reliable measurement of glucose, in a similar manner to conventional implantable sensors. However, from the perspective of the application of CGMS devices, the MN-based electrodes have certain drawbacks. One is a common problem of implantable sensors, that is, the loss of the sensitivity owing to surface fouling and enzyme inactivation induced by proteins and immune response [113-115]. This can be an obstacle in the use of a CGMS device in the current situation, as a longer period is required for glucose monitoring as commercially available devices achieving up to 14 days of monitoring of blood glucose [21]. Additionally, measurement methodologies of biological analytes, in the use of MNs as in vivo sensing probes, are basically limited to the enzymatic electrochemical measurement. This is because such MN-based electrode sensors are compatible not with complicated measurement systems but with relatively simple sensing principle such as electrochemical reactions at the surface of MNs. The limited measurement methodology, using only electrochemical sensing with conventional electrodes, restricts the use of more accurate methods of measurements such as FET-based sensors [116-118], which results in a limited number of measurable biomarkers.

On the other hand, the use of MNs as biological fluid collectors have an advantage over their use as sensing probes because of the developed technology of ex vivo analysis of extracted biological samples. In particular, pTAS devices meet the purpose of the MN-based fluid collector by achieving a small amount of direct flow of ISF or blood from inside the skin to measurement systems. From this perspective, the hydrogel-forming swellable MNs have a disadvantage of the requirement of an additional step to separate the absorbed ISF from the MN array. As microfluidic CGMS devices composed of microchannels have already been proposed using infrared light [119], viscosity-sensitive cantilever [120, 121], and enzymatic electrochemical measurement [122-124], the integration of the fluid collecting MNs with these devices would substantiate minimally invasive CGMS devices. However, the fabrication of such MNs is relatively complicated because, in order to integrate a $\mathrm{MN}$ array as a fluid collector 
with CGMS devices, fluidic channels in MNs should be precisely connected to the microchannels in the device. Furthermore, the sampling rate of MNs is crucial for CGMS. As reported by Samant and Prausnitz, the rank order of the amount of ISF collected by MNs is as follows: hydrogel MN (0.0030 pL per MN (12 h insertion) $)<$ paper-based porous $\mathrm{MN}(0.0033 \mathrm{pL}$ per $\mathrm{MN}$ (20 min insertion $))<$ hollow $\mathrm{MN}(0.01-0.03$ pL per MN (20 min insertion)) [111]. According to this analysis, hollow MNs enable sufficient extraction of ISF while the porous $\mathrm{MN}$ sampling rate is limited by a diffusion through the dermis. However, the porous MN provides advantages such as its applicability for biodegradable materials including PEG and the simple fabrication and assembly with microfluidic systems, whereas the hollow MN must be made of non-biocompatible materials and fabricated by a complex process which limits the microfluidic system design.

\section{Conclusion}

In this paper, the applicabilities of functionalized $\mathrm{MNs}$ to CGMS were discussed. The MN-based in vivo sensors have been applied to diagnostic systems even in a clinical experiment level, by being functionalized as electrodes, especially for glucose measurement. However, the MNs as sensing probes have been facing the challenges similar to those of the implantable sensors, such as the impaired sensitivity and the limited measurement methodology. On the other hand, the MN-based trans-dermal fluidic channels can be highlighted with the potential to be combined with recently developed pTAS technologies. Therefore, the use of MNs as biological fluid collectors should be investigated for integration with the existing ex vivo diagnostic systems and for a more efficient sampling mechanism of ISF. The development of functionalized MNs in those ways will contribute to the realization of commercially available CGMS devices in a minimally invasive manner instead of conventional glucose monitoring devices with pain.

\section{Authors' contributions}

$\mathrm{K} T$ reviewed, analyzed and wrote the paper, and BK supervised the overall work, and all authors provided comments and agreed with the final form of the manuscript. Both authors read and approved the final manuscript.

\section{Competing interests}

The authors declare that they have no competing interests.

\section{Funding}

This research was partially supported by Collaborative research project K29144 in IIS, the University of Tokyo.

\section{Publisher's Note}

Springer Nature remains neutral with regard to jurisdictional claims in published maps and institutional affiliations.
Received: 23 August 2018 Accepted: 26 September 2018

Published online: 24 October 2018

\section{References}

1. L. Lin, A.P. Pisano, Silicon-processed microneedles. J. Microelectromech. Syst. 8(1), 78-84 (1999)

2. P. Griss, G. Stemme, Novel, side opened out-of-plane microneedles for microfluidic transdermal interfacing, in Technical Digest MEMS 2002 IEEE international conference. Fifteenth IEEE International Conference on Micro Electro Mechanical Systems (Cat. No.02CH66), pp. 467-470 (2002)

3. P. Griss, G. Stemme, Side-opened out-of-plane microneedles for microfluidic transdermal liquid transfer. J. Microelectromech. Syst. 12(3), 296 (2003)

4. K. Kabseog, S.P. Daniel, M.L. Hong, C. Wooseong, K. Kyunghwan, L. Jeong-Bong, H.A. Chong, A tapered hollow metallic microneedle array using backside exposure of su-8. J. Micromech. Microeng. 14(4), 597 (2004)

5. A. Trautmann, P. Ruther, O. Paul, Microneedle arrays fabricated using suspended etch mask technology combined with fluidic through wafer vias, in IEEE the sixteenth annual international conference on micro electro mechanical systems. MEMS-03 Kyoto, pp. 682-685 (2003)

6. A. Trautmann, F. Heuck, R. Denfeld, P. Ruther, O. Paul, Detachable silicon microneedle stamps for allergy skin prick testing, in 19th IEEE international conference on micro electro mechanical systems, pp. 434-437 (2006)

7. Wei Chen, Rui Tian, Xu Can, Bryant C. Yung, Guohao Wang, Yijing Liu, Qianqian Ni, Fuwu Zhang, Zijian Zhou, Jingjing Wang, Gang Niu, Ying $\mathrm{Ma}$, Fu Liwu, Xiaoyuan Chen, Microneedle-array patches loaded with dual mineralized protein/peptide particles for type 2 diabetes therapy. Nat. Commun. 8(1), 1777 (2017)

8. L. Luca, G.R. Bertrand, D. Floriant, M. Frank, M.T. Rex, H.G. Richard, I. Adelina, Non-invasive, transdermal, path-selective and specific glucose monitoring via a graphene-based platform. Nat. Nanotechnol. 13(6), 504-511 (2018)

9. R.P. Mark, Microneedles for transdermal drug delivery. Adv. Drug Deliv. Rev. 56(5), 581-587 (2004)

10. M. van der Koen, J. Wim, B. Joke, Microneedle technologies for (trans) dermal drug and vaccine delivery. J. Control. Release 161 (2), 645-655 (2012)

11. W. Sun, Z. Araci, M. Inayathullah, S. Manickam, X. Zhang, A. Marc, M. Bruce, P. Marinkovich, A.T. Lane, C. Milla, J. Rajadas, M.J. Butte, Polyvinylpyrrolidone microneedles enable delivery of intact proteins for diagnostic and therapeutic applications. Acta Biomater. 9(8), 7767 (2013)

12. P. Van Damme, F. Oosterhuis-Kafeja, M. Van der Wielen, Y. Almagor, O. Sharon, Y. Levin, Safety and efficacy of a novel microneedle device for dose sparing intradermal influenza vaccination in healthy adults. Vaccine 27(3), 454-459 (2009)

13. M. Kim, H. Yang, H. Kim, H. Jung, H. Jung, Novel cosmetic patches for wrinkle improvement: retinyl retinoate- and ascorbic acid-loaded dissolving microneedles. Int. J. Cosmet. Sci. 36(3), 207-212 (2014)

14. P. Yonghun, J. Park, G.S. Chu, K.S. Kim, J.H. Sung, B. Kim, Transdermal delivery of cosmetic ingredients using dissolving polymer microneedle arrays. Biotechnol. Bioprocess Eng. 20(3), 543-549 (2015)

15. M. Karen, J.C. McElnay, F.D. Ryan, Children's views on microneedle use as an alternative to blood sampling for patient monitoring. Int. J. Pharm. Pract. 22(5), 335 (2013)

16. N. Fogh-Andersen, B.M. Altura, B.T. Altura, O. Siggaard-Andersen, Composition of interstitial fluid. Clin. Chem. 41(10), 1522-1525 (1995)

17. J.P. Le Floch, B. Bauduceau, M. Lévy, H. Mosnier-Pudar, C. Sachon, B. Kakou, Self-monitoring of blood glucose, cutaneous finger injury, and sensory loss in diabetic patients. Diabetes Care 31(10), e73-e73 (2008)

18. S. Vaddiraju, D.J. Burgess, I. Tomazos, F.C. Jain, F. Papadimitrakopoulos, Technologies for continuous glucose monitoring: current problems and future promises. J. Diab. Sci. Technol. 4(6), 1540-1562 (2010)

19. V. Lodwig, B. Kulzer, O. Schnell, L. Heinemann, Current trends in continuous glucose monitoring. J. Diab. Sci. Technol. 8(2), 390 (2014) 
20. R. David, Continuous glucose monitoring: a review of successes, challenges, and opportunities. Diab. Technol. Ther. 18(S2), S2-S13 (2016)

21. M.J. Fokkert, P.R. van Dijk, M.A. Edens, S. Abbes, D. de Jong, R.J. Slingerland, H.J.G. Bilo, Performance of the freestyle libre flash glucose monitoring system in patients with type 1 and 2 diabetes mellitus. BMJ Open 5(1), e000320 (2017)

22. S.B. Michael, David, K. Joy, D.S. Christopher, Timing of changes in interstitial and venous blood glucose measured with a continuous subcutaneous glucose sensor. Diabetes 52(II), 2790-2794 (2003)

23. J. Pickup, Developing glucose sensors for in vivo use. Trends Biotechnol. 11(7), 285-291 (1993)

24. Y. Miyahara, T. Moriizumi, K. Ichimura, Integrated enzyme fets for simultaneous detections of urea and glucose. Sens. Actuat. 7(1), 1-10 (1985)

25. H. Yoon, Sungrok Ko, J. Jang, Field-effect-transistor sensor based on enzyme-functionalized polypyrrole nanotubes for glucose detection. J. Phys. Chem. B 112(32), 9992-9997 (2008)

26. J. Kimura, N. Ito, T. Kuriyama, M. Kikuchi, T. Arai, N. Negishi, Y. Tomita, A novel blood glucose monitoring method an isfet biosensor applied to transcutaneous effusion fluid. J. Electrochem. Soc. 136(6), 1744-1747 (1989)

27. P. Keun-Yong, C. Sang-Bok, L. Minho, S. Byung-Ki, C. Sie-Young, Isfet glucose sensor system with fast recovery characteristics by employing electrolysis. Sens. Actuators 83(1), 90-97 (2002)

28. M.R. Robinson, R.P. Eaton, D.M. Haaland, G.W. Koepp, E.V. Thomas, B.R. Stallard, P.L. Robinson, Noninvasive glucose monitoring in diabetic patients: a preliminary evaluation. Clin. Chem. 38(9), 1618-1622 (1992)

29. T. Andrea, M. Alberto, P. Giovanni, Non-invasive glucose monitoring: assessment of technologies and devices according to quantitative criteria. Diabetes Res. Clin. Pract. 77(1), 16-40 (2007)

30. F.D. Ryan, M. Karen, E. Caffarel-Salvador, B.M. Torrisi, E. Eyman, J.C. McElnay, Microneedle-mediated minimally invasive patient monitoring. Ther. Drug Monit. 36(1), 10-17 (2014)

31. P.R. Miller, R.J. Narayan, R. Polsky, Microneedle-based sensors for medical diagnosis. J. Mater. Chem. B 4, 1 (2016)

32. V. Letizia, M.S. Lucanos, B. Giuseppe, Microneedles for transdermal biosensing: current picture and future direction. Adv. Healthcare Mater. 4(17), 2606-2640 (2015)

33. S.J. Updike, M.C. Shults, B.J. Gilligan, R.K. Rhodes, A subcutaneous glucose sensor with improved longevity, dynamic range, and stability of calibration. Diabetes Care 23(2), 208-214 (2000)

34. P. Jung-Hwan, M.G. Allen, M.R. Prausnitz, Biodegradable polymer microneedles: fabrication, mechanics and transdermal drug delivery. J. Control. Release 104(1), 51-66 (2005)

35. J.Y. Lee, K. Dae-Hyun, K.J. Lee II, H. Seo, S.H. Park, E.H. Jang, Y. Park, Y.-N. Youn, W. Ryu, Transfer-molded wrappable microneedle meshes for perivascular drug delivery. J. Control. Release 268, 237-246 (2017)

36. Y. Ito, K. Matsumoto, N. Osakama, R. Yoshioka, S. Kobuchi, T. Sakaeda, K. Takada, Dissolving microneedles as skin allergy test device. Biol. Pharm. Bull. 40(4), 531-534 (2017)

37. K. Tsioris, W.K. Raja, E.M. Pritchard, B. Panilaitis, D.L. Kaplan, F.G. Omenetto, Fabrication of silk microneedles for controlled-release drug delivery. Adv. Funct. Mater. 22(2), 330 (2012)

38. J.D. Kim, M. Kim, H. Yang, K. Lee, H. Jung, Droplet-born air blowing: nove dissolving microneedle fabrication. J. Control. Release 170(3), 430-436 (2013)

39. K.J. Lee, S.H. Park, J.Y. Lee, H.C. Joo, E.H. Jang, Y.N. Youn, W. Ryu, Perivascular biodegradable microneedle cuff for reduction of neointima formation after vascular injury. J. Control. Release 192(Supplement C), 174-181 (2014)

40. S.J. Lee, H.S. Yoon, X. Xuan, J.Y. Park, S.J. Paik, M.G. Allen, A patch type non-enzymatic biosensor based on $3 \mathrm{~d}$ sus micro-needle electrode array for minimally invasive continuous glucose monitoring. Sens. Actuat. B 222, 1144-1151 (2016)

41. Y. Sun, L. Ren, L. Jiang, Y. Tang, B. Liu, Fabrication of composite microneedle array electrode for temperature and bio-signal monitoring. Sensors 18(4), 1193 (2018)

42. S.A. Skoog, P.R. Miller, R.D. Boehm, A.V. Sumant, R. Polsky, R.J. Narayan, Nitrogen-incorporated ultrananocrystalline diamond microneedle arrays for electrochemical biosensing. Diamond Related Mater. $\mathbf{5 4}$ 39-46 (2015)
43. L. Tang, Y. Li, H. Xie, Q. Shu, F. Yang, Y. Liu, F. Liang, H. Wang, Weihua Huang, G. Zhang, A sensitive acupuncture needle microsensor for realtime monitoring of nitric oxide in acupoints of rats. Sci. Rep. 7(1), 6446 (2017)

44. D. Chen, C. Wang, W. Chen, Y. Chen, X.J. John, Pvdf-nafion nanomembranes coated microneedles for in vivo transcutaneous implantable glucose sensing. Biosens. Bioelectron. 74, 1047-1052 (2015)

45. Z. Jin-Xiu, T. Li-Na, Y. Fan, L. Feng-Xia, W. Hua, L. Yu-Tao, Z. Guo-Jun, Mos2/pt nanocomposite-functionalized microneedle for real-time monitoring of hydrogen peroxide release from living cells. Analyst 142, 4 (2017)

46. S.R. Chinnadayyala, I. Park, S. Cho, Nonenzymatic determination of glucose at near neutral ph values based on the use of nafion and platinum black coated microneedle electrode array. Microchimica Acta 185(5), 250 (2018)

47. L. Ren, Q. Jiang, Z. Chen, K. Chen, X. Shujia, J. Gao, L. Jiang, Flexible microneedle array electrode using magnetorheological drawing lithography for bio-signal monitoring. Sens. Actuators A 268, 38-45 (2017)

48. C. O'Mahony, K. Grygoryev, A. Ciarlone, G. Giannoni, A. Kenthao, P. Galvin, Design, fabrication and skin-electrode contact analysis of polymer microneedle-based ecg electrodes. J. Micromech. Microeng. 26(8), 084005 (2016)

49. K. Chen, L. Ren, Z. Chen, C. Pan, W. Zhou, L. Jiang, Fabrication of microneedle electrodes for bio-signal recording by a magnetization-induced self-assembly method. Sensors 16(9), 1533 (2016)

50. G. Stavrinidis, K. Michelakis, V. Kontomitrou, G. Giannakakis, M. Sevrisarianos, G. Sevrisarianos, N. Chaniotakis, Y. Alifragis, G. Konstantinidis, Su-8 microneedles based dry electrodes for electroencephalogram. Microelectr. Eng. 159, 114-120 (2016)

51. S. Sharma, Z. Huang, M. Rogers, M. Boutelle, A.E.G. Cass, Evaluation of a minimally invasive glucose biosensor for continuous tissue monitoring. Anal. Bioanal. Chem. 408(29), 8427-8435 (2016)

52. S. Sharma, A. El-Laboudi, M. Reddy, N. Jugnee, S. Sivasubramaniyam, M. El Sharkawy, P. Georgiou, D. Johnston, N. Oliver, E.G. Anthony, A pilot study in humans of microneedle sensor arrays for continuous glucose monitoring. Anal. Methods 10, 2088-2095 (2018)

53. A.E.G. Cass, S. Sharma, Chapter fifteen - microneedle enzyme sensor arrays for continuous in vivo monitoring, in Enzymes as sensors of methods in enzymology, vol. 589, ed. by R.B. Thompson, C.A. Fierke (Academic Press, New York, 2017), pp. 413-427

54. A. McConville, J. Davis, Transdermal microneedle sensor arrays based on palladium: polymer composites. Electrochem. Commun. 72, 162-165 (2016)

55. P. Dardano, A. Calio, J. Politi, V. Di Palma, M.F. Bevilacqua, I. Rea, M. Casalino, A. Di Matteo, I. Rendina, L. De Stefano, Hybrid microneedles devices for diagnostic and therapeutic applications: fabrication and preliminary results. Int. Soc. Optics Phot. 9518, 95180 (2015)

56. P. Dardano, A. Calio, J. Politi, I. Rea, L. De Stefano, V. Di Palma, M. F. Bevilacqua, A. Di Matteo, Diagnostic and therapeutic devices based on polymeric microneedles: fabrication and preliminary results, in 2015 XVIII AISEM annual conference, pp. 1-4 (2015)

57. C. Barrett, K. Dawson, C. O'Mahony, A. O'Riordan, Development of low cost rapid fabrication of sharp polymer microneedles for in vivo glucose biosensing applications. ECS J. Solid State Sci. Technol. 4(10), S3-S8 (2015)

58. S. Sharma, E. Takagi, T. Cass, W. Tsugawa, K. Sode, Minimally invasive microneedle array electrodes employing direct electron transfer type glucose dehydrogenase for the development of continuous glucose monitoring sensors. Procedia Technol. 27, 208-209 (2017)

59. K.D. Hee, J.H. Sang, W. Taejun, S.M. Hwan, K. Young-Eun, K.K. Hean, A. One, H.S. Kwang, Microneedle biosensor for real-time electrical detection of nitric oxide for in situ cancer diagnosis during endomicroscopy. Adv. Healthcare Mater. 4(8), 1153-1158 (2015)

60. S. Sharma, A. Saeed, C. Johnson, N. Gadegaard, A.E.G. Cass, Rapid, low cost prototyping of transdermal devices for personal healthcare monitoring. Sens. Bio-Sens. Res. 13, 104-108 (2017)

61. J.R. Windmiller, G. Valdes-Ramirez, N. Zhou, M. Zhou, P.R. Miller, C. Jin, S.M. Brozik, R. Polsky, E. Katz, R. Narayan, J. Wang, Bicomponent microneedle array biosensor for minimally-invasive glutamate monitoring. Electroanalysis 23(10), 2 (2011) 
62. G. Valdes-Ramfrez, Y.-C. Li, J. Kim, W. Jia, A.J. Bandodkar, R. Nunez-Flores, P.R. Miller, W. Shu-Yii, R. Narayan, J.R. Windmiller, R. Polsky, J. Wang, Microneedle-based self-powered glucose sensor. Electrochem. Commun. 47, 58-62 (2014)

63. K. Rupesh, A.M. Mishra, M. Vinu, S. Fernando, C. Robert, W. Joseph, A microneedle biosensor for minimally-invasive transdermal detection of nerve agents. Analyst 142, 918-924 (2017)

64. A.M. Vinu, Mohan, Joshua Ray Windmiller, Rupesh K. Mishra, and Joseph Wang. Continuous minimally-invasive alcohol monitoring using microneedle sensor arrays. Biosens. Bioelectron. 91, 574-579 (2017)

65. C. Bianca, M. Aida, M. Rupesh, B. Barbara, N. Tatsuo, D. Thomas, L. Mengjia, C. Cecilia, S. Robert, W. Joseph, Wearable wireless tyrosinase bandage and microneedle sensors: toward melanoma screening. Adv. Healthcare Mater. 7(7), 1701264 (2018)

66. F. Ribet, G. Stemme, N Roxhed, Microneedle-based system for minimally invasive continuous monitoring of glucose in the dermal interstitial fluid, in 2018 IEEE micro electro mechanical systems (MEMS), pp. 408-411 (2018)

67. N. Vasylieva, S. Marinesco, D. Barbier, A. Sabac, Silicon/su8 multielectrode micro-needle for in vivo neurochemical monitoring. Biosens. Bioelectron. 72, 148-155 (2015)

68. F. Barz, P. Ruther, S. Takeuchi, O. Paul, Flexible silicon-polymer neural probe rigidified by dissolvable insertion vehicle for high-resolution neural recording with improved duration, in 2015 28th IEEE international conference on micro electro mechanical systems (MEMS), pp. 636-639 (2015)

69. K. Seidl, S. Herwik, T. Torfs, H.P. Neves, O. Paul, P. Ruther, Cmos-based high-density silicon microprobe arrays for electronic depth control in intracortical neural recording. J. Microelectromech. Syst. 20(6), 1439-1448 (2011)

70. P. R, Stanislav Herwik, S. Kisban, K. Seidl, O. Paul, Recent progress in neural probes using silicon mems technology. IEEJ Trans. Elect. Electr. Eng. 5(5), 505-515 (2010)

71. S. Herwik, S. Kisban, A.A.A. Aarts, K. Seidl, G. Girardeau, K. Benchenane, M.B. Zugaro, S.I. Wiener, O. Paul, H.P. Neves, P. Ruther, Fabrication technology for silicon-based microprobe arrays used in acute and subchronic neural recording. J. Micromech. Microeng. 19(7), 074008 (2009)

72. P. Parikh, H. Mochari, L. Mosca, Clinical utility of a fingerstick technology to identify individuals with abnormal blood lipids and high-sensitivity c-reactive protein levels. Am. J. Health Promot. 23(4), 279-282 (2009)

73. S.K. Garg, R.O. Potts, N.R. Ackerman, S.J. Fermi, J.A. Tamada, H.P. Chase, Correlation of fingerstick blood glucose measurements with glucowatch biographer glucose results in young subjects with type 1 diabetes. Diabetes Care 22(10), 1708-1714 (1999)

74. D. Loewenstein, C. Stake, M. Cichon, Assessment of using fingerstick blood sample with i-stat point-of-care device for cardiac troponin i assay. Am. J. Emerg. Med. 31(8), 1236-1239 (2013)

75. S.D. Gittard, A. Ovsianikov, B.N. Chichkov, A. Doraiswamy, R.J. Narayan, Two-photon polymerization of microneedles for transdermal drug delivery. Expert Opin. Drug Delivery 7(4), 513-533 (2010)

76. R. Liu, X. Wang, Y. Feng, G. Wang, J. Liu, H. Ding, Theoretical analytical flow model in hollow microneedles for non-forced fluid extraction, in 2006 1st IEEE international conference on nano/micro engineered and molecular systems, pp. 1039-1042 (2006)

77. C.G. Li, C.Y. Lee, K. Lee, H. Jung, An optimized hollow microneedle for minimally invasive blood extraction. Biomed. Microdevices 15(1), 17-25 (2013)

78. H.L. Thanh, B.Q. Ta, H.L. The, V. Nguyen, K. Wang, F. Karlsen, Low-cost fabrication of hollow microneedle arrays using $\mathrm{cnc}$ machining and uv lithography. J. Microelectromech. Syst. 24(5), 1583-1593 (2015)

79. C.G. Li, M. Dangol, C.Y. Lee, M. Jang, H. Jung, A self-powered one-touch blood extraction system: a novel polymer-capped hollow microneedle integrated with a pre-vacuum actuator. Lab Chip 518, 15 (2015)

80. B.Q. Tran, P.R. Miller, R.M. Taylor, G. Boyd, P.M. Mach, C.N. Rosenzweig, J.T. Baca, R. Polsky, T. Glaros, Proteomic characterization of dermal interstitial fluid extracted using a novel microneedle-assisted technique. J. Proteome Res. 17(1), 479-485 (2018)

81. D.S. Lee, C.G. Li, C. Ihm, H. Jung, A three-dimensional and bevel-angled ultrahigh aspect ratio microneedle for minimally invasive and painless blood sampling. Sens. Actuators B 255, 384 (2018)
82. M. Philip, M. Matthew, M. Ron, A. Carlee, B. Igal, W. David, N. Roger, P. Ronen, Towards an integrated microneedle total analysis chip for protein detection. Electroanalysis $\mathbf{2 8}(6), 1$ (2016)

83. P.R. Miller, X. Xiao, I. Brener, D.B. Burckel, R. Narayan, R. Polsky, Microneedle-based transdermal sensor for on- chip potentiometric determination of k+. Adv Healthcare Mater. 3(6), 876-881 (2013)

84. S. Zimmermann, D. Fienbork, B. Stoeber, A.W. Flounders, D. Liepmann, A microneedle-based glucose monitor: fabricated on a wafer-level using in-device enzyme immobilization, in 12th international conference on TRANSDUCERS, Solid-State sensors, actuators and microsystems, 2003, vol 1, pp. 99-102 (2003)

85. E.V. Mukerjee, S.D. Collins, R.R. Isseroff, R.L. Smith, Microneedle array for transdermal biological fluid extraction and in situ analysis. Sens. Actuators A: Phys. 114(2), 267-275 (2004)

86. P.M. Wang, M. Cornwell, M.R. Prausnitz, Minimally invasive extraction of dermal interstitial fluid for glucose monitoring using microneedles. Diabetes Technol. Ther. 7(1), 131-141 (2005)

87. B. Chua, S.P. Desai, M.J. Tierney, J.A. Tamada, A.N. Jina, Effect of microneedles shape on skin penetration and minimally invasive continuous glucose monitoring in vivo. Sens. Actuators A 203, 373 (2013)

88. L.M. Strambini, A. Longo, S. Scarano, T. Prescimone, I. Palchetti, M. Minunni, D. Giannessi, G. Barillaro, Self-powered microneedle-based biosensors for pain-free high-accuracy measurement of glycaemia in interstitial fluid. Biosens. Bioelectron. 66, 162-168 (2015)

89. C.G. Li, H.-A. Joung, H. Noh, M.-B. Song, M.-G. Kim, H. Jung, One-touchactivated blood multidiagnostic system using a minimally invasive hollow microneedle integrated with a paper-based sensor. Lab Chip 15 6 (2015)

90. D. Nicholas, K.A. Logan, Y. Sheng, J. Gao, S. Farrell, D. Dixon, B. Callan, A.P. McHale, J.F. Callan, Rapid paper based colorimetric detection of glucose using a hollow microneedle device. Int. J. Pharm. 547(1), 244-249 (2018)

91. L.M. Yu, F.E.H. Tay, D.G. Guo, L. Xu, K.L. Yap, A microfabricated electrode with hollow microneedles for ECG measurement. Sens. Actuators, A 151(1), 17-22 (2009)

92. S.A. Ranamukhaarachchi, C. Padeste, M. Diibner, U.O. Hafeli, B. Stoeber, V.J. Cadarso, Integrated hollow microneedle-optofluidic biosensor for therapeutic drug monitoring in sub-nanoliter volumes. Sci. Rep. $\mathbf{6}$, 29075 (2016)

93. S.A. Ranamukhaarachchi, C. Padeste, U.O. Hafeli, B. Stoeber, V.J. Cadarso, Design considerations of a hollow microneedle-optofluidic biosensing platform incorporating enzyme-linked assays. J. Micromech. Microeng. 28(2), 024002 (2018)

94. Andrey V. Romanyuk, Vasiliy N. Zvezdin, Pradnya Samant, Mark L. Grenader, Marina Zemlyanova, Mark R. Prausnitz, Collection of analytes from microneedle patches. Anal. Chem. 86(21), 10520-10523 (2014)

95. R.F. Donnelly, M.T.C. McCrudden, A.Z. Alkilani, E. Larraneta, E. McAlister, A.J. Courtenay, M.-C. Kearney, T.R.R. Singh, H.O. McCarthy, V.L. Kett, E. Caffarel-Salvador, S. Al-Zahrani, A.D. Woolfson, Hydrogel-forming microneedles prepared from "super swelling" polymers combined with lyophilised wafers for transdermal drug delivery. PLoS ONE. 9(10), 1-12 (2014)

96. E. CafFarel-Salvador, A.J. Brady, E. Eltayib, T. Meng, A. Alonso-Vicente, P. Gonzalez-Vazquez, B.M. Torrisi, E.M. Vicente-Perez, K. Mooney, D.S. Jones, S.E.J. Bell, C.P. McCoy, H.O. McCarthy, J.C. McElnay, R.F. Donnelly, Hydrogel-forming microneedle arrays allow detection of drugs and glucose in vivo: potential for use in diagnosis and therapeutic drug monitoring. PLoS ONE. 10(12), 1-21 (2016)

97. H. Chang, M. Zheng, X. Yu, A. Than, R.Z. Seeni, R. Kang, J. Tian, D.P. Khanh, L. Liu, P. Chen, C. Xu, A swellable microneedle patch to rapidly extract skin interstitial fluid for timely metabolic analysis. Adv. Mater. 29(37), 1702243 (2017)

98. MinYoung Kim, Bokyung Jung, Jung-Hwan Park, Hydrogel swelling as a trigger to release biodegradable polymer microneedles in skin. Biomaterials 33(2), 668-678 (2012)

99. R.F. Donnelly, T. Raghu, R. Singh, A.Z. Alkilani, M.T.C. McCrudden, S. O'Neill, C. O'Mahony, K. Armstrong, N. McLoone, P. Kole, A.D. Woolfson, Hydrogel-forming microneedle arrays exhibit antimicrobial properties: potential for enhanced patient safety. Int. J. Pharm. 451(1), 76-91 (2013)

100. R.F. Donnelly, K. Mooney, M.T.C. Mccrudden, E.M. Vicente-Perez, L. Belaid, P. Gonzalez-Vazquez, J.C. Mcelnay, A.D. Woolfson, Hydrogel-forming 
microneedles increase in volume during swelling in skin, but skin barrier function recovery is unaffected. J. Pharm. Sci. 103(5), 1478-1486 (2014)

101. J.G. Hardy, E. Larraneta, R.F. Donnelly, N. McGoldrick, K. Migalska, M.T.C. McCrudden, N.J. Irwin, L. Donnelly, C.P. McCoy, Hydrogel-forming microneedle arrays made from light-responsive materials for ondemand transdermal drug delivery. Mol. Pharm. 13(3), 907-914 (2016)

102. J. Ji, F.E.H. Tay, J. Miao, C. Iliescu, Microfabricated microneedle with porous tip for drug delivery. J. Micromech. Microeng. 16(5), 958 (2006)

103. M. Shirkhanzadeh, Microneedles coated with porous calcium phosphate ceramics: effective vehicles for transdermal delivery of solid trehalose. J. Mater. Sci. 16(1), 37-45 (2005)

104. J.-H. Park, S.-O. Choi, R. Kamath, Y.-K. Yoon, M.G. Allen, M.R. Prausnitz, Polymer particle-based micromolding to fabricate novel microstructures. Biomed. Microdev. 9(2), 223-234 (2007)

105. K. van der Maaden, R. Luttge, P. Jan Vos, J. Bouwstra, G. Kersten, I Ploemen. Microneedle-based drug and vaccine delivery via nanoporous microneedle arrays. Drug Deliv. Transl. Res. 5(4), 406 (2015)

106. J. Li, B. Liu, Y. Zhou, Z. Chen, L. Jiang, W. Yuan, L. Liang, Fabrication of a ti porous microneedle array by metal injection molding for transdermal drug delivery. PLOS ONE 12(2), 1-15 (2017)

107. Laith Humrez, Maximiano Ramos, Ahmed Al-Jumaily, Mira Petchu, John Ingram, Synthesis and characterisation of porous polymer microneedles. J. Polym. Res. 18(5), 1043-1052 (2011)

108. M. Verhoeven, S. Bystrova, L. Winnubst, H. Qureshi, T.D. de Gruijl, R.J. Scheper, R. Luttge, Applying ceramic nanoporous microneedle arrays as a transport interface in egg plants and an ex vivo human skin model. Microelect. Eng. 98, 659-662 (2012)

109. Liming Liu, Hiroyuki Kai, Kuniaki Nagamine, Yudai Ogawa, Matsuhiko Nishizawa, Porous polymer microneedles with interconnecting microchannels for rapid fluid transport. RSC Adv. 6, 48630-48635 (2016)

110. Kuniaki Nagamine, Jun Kubota, Hiroyuki Kai, Yoshinobu Ono, Matsuhiko Nishizawa, An array of porous microneedles for transdermal monitoring of intercellular swelling. Biomed. Microdev. 19(3), 68 (2017)

111. Pradnya P. Samant, Mark R. Prausnitz, Mechanisms of sampling interstitial fluid from skin using a microneedle patch. Proc. Natl. Acad. Sci. 115(18), 4583-4588 (2018)

112. K. Takeuchi, B. Kim, K. Sharma, P. Ruther, O. Paul, Microfluidic chip for glucose monitoring with biodegradable microneedles, in 5th international conference on microneedles. 2018, pp. 101

113. W.K. Ward, J.E. Troupe, Assessment of chronically implanted subcutaneous glucose sensors in dogs: the effect of surrounding fluid masses. ASAIO J. 45(6), 555-561 (1999)
114. R.M. Rhemrev-Boom, R.G. Tiessen, A.A. Jonker, K. Venema, P. Vadgama, J. Korf, A lightweight measuring device for the continuous in vivo monitoring of glucose by means of ultraslow microdialysis in combination with a miniaturised flow-through biosensor. Clin. Chim. Acta. 1, 1-10 (2002)

115. M. Gerritsen, J.A. Jansen, A. Kros, D.M. Vriezema, N.A.J.M. Sommerdijk, R.J.M. Nolte, J.A. Lutterman, S.W.F.M. Van Hovell, A. Van der Gaag, Influence of inflammatory cells and serum on the performance of implantable glucose sensors. J. Biomed. Mater. Res. 54(1), 69-75 (2000)

116. Taira Kajisa, Toshiya Sakata, Glucose-responsive hydrogel electrode for biocompatible glucose transistor. Sci. Technol. Adv. Mater. 18(1), 26-33 (2017)

117. Akira Matsumoto, Naoko Sato, Toshiya Sakata, Kazunori Kataoka, Yuji Miyahara, Glucose-sensitive field effect transistor using totally synthetic compounds. J. Solid State Electrochem. 13(1), 165-170 (2009)

118. J. Liu, M. Agarwal, K. Varahramyan, Glucose sensor based on organic thin film transistor using glucose oxidase and conducting polymer. Sensors Actuators B 135(1), 195-199 (2008)

119. B.U. Moon, M.G. de Vries, B.H.C. Westerink, E. Verpoorte, Development and characterization of a microfluidic glucose sensing system based on an enzymatic microreactor and chemiluminescence detection. Sci. China Chem. 55(4), 515-523 (2012)

120. Yongjun Zhao, Siqi Li, Arthur Davidson, Bozhi Yang, Qian Wang, Qiao Lin, A mems viscometric sensor for continuous glucose monitoring. J. Micromech. Microeng. 17(12), 2528 (2007)

121. Xian Huang, Siqi Li, Jerome S. Schultz, Qian Wang, Qiao Lin, A mems affinity glucose sensor using a biocompatible glucose-responsive polymer. Sensors Actuators B 140(2), 603-609 (2009)

122. G. Piechotta, J. Albers, R. Hintsche, Novel micromachined silicon sensor for continuous glucose monitoring. Biosens. Bioelectron. 21(5), 802-808 (2005)

123. C.-J. Huang, Y.-H. Chen, C.-H. Wang, T.-C. Chou, G.-B. Lee, Integrated microfluidic systems for automatic glucose sensing and insulin injection. Sensors Actuators B. 122(2), 461-468 (2007)

124. Pu Zhihua, Chongwei Zou, Ridong Wang, Xiaochen Lai, Yu. Haixia, Xu Kexin, Dachao Li, A continuous glucose monitoring device by graphene modified electrochemical sensor in microfluidic system. Biomicrofluidics 10(1), 011910 (2016)

\section{Submit your manuscript to a SpringerOpen ${ }^{\circ}$ journal and benefit from:}

- Convenient online submission

- Rigorous peer review

- Open access: articles freely available online

- High visibility within the field

- Retaining the copyright to your article

Submit your next manuscript at springeropen.com 